\title{
Entrelacs
}

ENTRELACS Cinéma et audiovisuel

$11 \mid 2014$

La Voix

\section{La voix entre excès et retrait : les fantômes de l'opéra dans le cinéma muet}

Sophie Herr

\section{(2) OpenEdition}

Journals

Édition électronique

URL : http://journals.openedition.org/entrelacs/1422

DOI : 10.4000 /entrelacs. 1422

ISSN : 2261-5482

Éditeur

Éditions Téraèdre

Référence électronique

Sophie Herr, "La voix entre excès et retrait : les fantômes de l'opéra dans le cinéma muet », Entrelacs [En ligne], 11 | 2014, mis en ligne le 07 janvier 2015, consulté le 30 avril 2019. URL : http:// journals.openedition.org/entrelacs/1422 ; DOI : 10.4000/entrelacs. 1422

Ce document a été généré automatiquement le 30 avril 2019

Tous droits réservés 


\title{
La voix entre excès et retrait : les fantômes de l'opéra dans le cinéma muet
}

\author{
Sophie Herr
}

\section{Introduction - Farrar voiceless!}

1 «La Farrar sans voix ! " ${ }^{1}$ 'exclame Photoplay Magazine au moins d'août 1915 en réaction à l'annonce de la participation de la chanteuse d'opéra dans une nouvelle production des studios Lasky à Hollywood. La presse ne manque pas de relever le paradoxe de la diva muette qui tels «la Mona Lisa sans son sourire, un stradivarius sans ses cordes, des enfants d'un institut de sourds-muets se produisant au théâtre " perd l'essentiel de ses attributs. Que reste-t-il de la présence vibrante de la diva, de sa voix adorée du public, si l'industrie cinématographique commercialise une ombre silencieuse mécaniquement projetée dans les salles de cinéma? Le cinématographe dérobe ainsi au public l'élément vocal à travers lequel s'est tissé un lien affectif avec la chanteuse. Comment le médium cinématographique peut-il combler ce manque ? Que reste-t-il de la voix lyrique, de ses qualités esthétiques, de sa puissance et de son pouvoir d'attraction dans un médium tel que le cinéma muet?

2 Si l'opéra célèbre la puissance affective et esthétique de la voix tout comme sa virtuosité technique, le film transpose le monde sonore à l'image muette. Tandis qu'à l'opéra la voix " désincarnée $~^{2}$ de la diva triomphe de la réalité triviale du corps qu'elle transcende, au cinéma, ce sont les mouvements expressifs de la parole, la gestuelle et la « lisibilité » du corps de l'acteur qui compensent l'absence de voix. Cependant, si celui-ci a ouvert aux dieux du monde lyrique un espace médial supplémentaire, c'est en renouant avec la splendeur de leur art vocal. De quelle mise en scène médiale et de quelle transposition intermédiale la voix lyrique fait-elle l'objet au cinéma ? Dans quelle mesure les figurations cinématographiques de la voix héritent-elles de la puissance esthétique et 
affective du chant lyrique ? Comment le cinéma muet peut-il, malgré - ou grâce à - l' absence de la voix se référer à l'art lyrique?

3 L'opéra et le cinéma se rejoignent certes dans le projet esthétique du concours des arts énoncé par le Gesamtkunstwerk wagnérien ${ }^{3}$, mais rien n'oppose davantage le nouveau médium muet à l'art lyrique qui, dans sa tonalité italienne, est centré sur la magnificence du chant. C'est pourtant précisément autour de l'élément vocal que se nouent les points de rencontre entre cinéma et opéra, comme je tenterai de le montrer. A cette fin, j'interrogerai la mise en scène de la voix d'opéra en dégageant quelques aspects exemplaires tels la normativité du "beau chant", son caractère de "costume», son rapport au geste ainsi que la construction esthétique de la diva « muette ». Il s'agit de voir comment la mise en scène de la voix muette peut influencer l'imagination spectatorielle et mobilise un champ ouvert d'intervocalités. Réfléchissant le rapport du chant à l'image, la voix d'opéra apparaît comme l'autre face du miroir et du symbolique, sous la forme d'un fantôme. Finalement, il s'agira de dégager la dimension réflexive de la figuration de la voix lyrique comprise à la fois comme mise en scène de la réflexivité propre à la voix et comme réflexion des possibilités même du médium. Je tenterai de montrer à travers quelques exemples de films paradigmatiques - The Phantom of the Opera de Rupert Julian (1925), Die Löwenbraut de Max Obal (1913-14), My Cousin d'Edward José (1918) et The Mysterious Lady de Fred Niblo (1928) - comment il revient à la «mutité» de la voix de conférer au cinéma son caractère « opératique ».

\section{L'attirance du cinéma pour l'opéra}

4 Après sa première apparition au cinéma en 1915, Geraldine Farrar sera à l'affiche de quatorze films en l'espace de cinq ans. Dès ses débuts, le cinéma s'inspire volontiers des sujets d'opéra ${ }^{4}$ : les adaptations de Carmen s'élèvent au nombre de soixante, tandis que les Tosca muettes célèbrent la rencontre et la légitimation réciproque du vérisme italien avec le cinéma. Les chanteurs immortalisent leurs grands rôles sur celluloïd s'ils ne figurent à l'écran en leur qualité même d'artiste lyrique, à l'exemple d'Enrico Caruso dans My Cousin (1918). La première vague d'opéras muets des années 1905-1907 est liée à la crise d'identité du nouveau médium qui va conduire au Film d'Art. Par l'exploitation de la figure de la diva d'opéra, l'industrie du cinéma entend profiter de la respectabilité sociale et culturelle du chanteur afin d'atteindre un public bourgeois et cultivé. Le recours aux motifs de l'opéra assure par ailleurs la familiarité du public avec l'argument narratif et les airs célèbres guident habilement l'imagination sonore.

5 Si la rencontre de l'opéra et du cinéma fait l'objet, en tant que genre artistique ${ }^{5}$, d'intenses discussions esthétiques, théoriques et pratiques, la définition de l'opéra est loin d'être uniforme et dépend des traditions musicales et théâtrales nationales. Contrairement à la France, où la construction de l'analogie opéra/cinéma se fonderait ${ }^{6}$ peut-être davantage sur le support dramatique ${ }^{7}$, en Allemagne, elle reposerait sur la réception de l'idée wagnérienne de l'œuvre d'art totale qui met l'accent sur la place attribuée à la musique dans la projection cinématographique ${ }^{8}$. Visant bien moins une synchronisation son-image réaliste ou naturaliste qu'un jeu créatif avec les pratiques de projection, elles se révèlent souvent être prétexte à l'exploitation de toutes sortes de procédés de synchronisation "vivante » ou de techniques mécaniques de sonorisation extra-cinématographiques'. En Italie, où la beauté et les effets de la voix chantée jouent un rôle central dans la définition du genre "opéra ", l'analogie entre opéra et cinéma 
s'oriente davantage à l'aura et à l'expressivité de ses interprètes, les divas. Comme si, celles-ci, reprenant le répertoire des gestes et des attitudes des divas d'opéra portaient à l'écran, même muettes, quelque chose de la force vocale de leurs modèles.

6 La question du caractère opératique du cinéma ne saurait se réduire au concours des arts, au rapport de thématisation intermédiale et aux phénomènes d'emprunt ou d'hybridation. J'envisagerai dans les lignes suivantes la dimension opératique ${ }^{10}$ du cinéma qui, se révélant de manière paradigmatique dans la figure de la diva muette, se comprend davantage comme une attirance des contraires qu'un effort de concordance des arts. Il s'agit alors de voir comment l'attirance du cinéma pour l'opéra qui se noue autour de l'élément vocal conduit à l'exploitation esthétique d'une différence médiale.

\section{Caruso : la voix du masque}

$7 \quad$ Il est difficile de juger de la familiarité du public de cinéma avec la voix de Caruso lorsque le chanteur apparaît sur les écrans dans My Cousin (1918), son premier film, dans lequel il interprète à la fois le rôle de Tommasso, un sculpteur sans-le-sou et de son cousin Caroli, un chanteur d'opéra célébrissime. Si les producteurs Lasky et Zukor visaient l'effet publicitaire d'un chanteur aussi célèbre que Caruso, la mise en scène de la voix d'opéra joue cependant moins sur le caractère personnel que sur une certaine physionomie de la voix culturellement formée et exercée. La mise en scène cinématographique de la voix exacerbe le contraste entre le «bel organe " travaillé de Caroli et la voix, certes authentique, à l'expression sincère mais exprimant le dénuement de toute maitrise technique de Tommasso. L'imagination des spectateurs est plus ou moins guidée par des reaction shots ${ }^{11}$ et par la mise en contraste de la mimique et de la gestuelle du « bon » et du «mauvais » chanteur. En s'ouvrant sur une succession de portraits du chanteur vêtu de costumes divers, le film annonce l'emploi multiple de Caruso: aux rôles jumeaux de Tommasso et Caroli s'ajoute son propre rôle de chanteur d'opéra à travers l'insertion filmique d'un fragment documentaire d'une représentation de Pagliacci de Leoncavallo au Metropolitan Opera - à savoir le célèbre air Vesti la giubba ${ }^{12}$ réfléchissant précisément le jeu actorial. Cette mise en abîme des jeux de rôle (du choix de casting jusqu'à la citation de l'opéra dans le film) atteint finalement son paroxysme dans l'élément même de la voix. À travers la mise en scène, les voix de Tommasso et Caroli, immédiatement reconnaissables à leurs traits typiques, apparaissent tels des masques de la commedia del arte, ou tels un «costume». Ainsi Theodor W. Adorno, qui voyait dans le costume le propre de l'opéra, considérait la voix elle-même comme « une partie du costume» ${ }^{13}$. En tant que représentation sonore du rôle, le « masque vocal » participe au jeu de masquage, démasquage, dédoublage, qui traverse l'ensemble du film ${ }^{14}$. Ce jeu de masques et de costumes se trouve étendu à ce que l'on pourrait appeler une projection sonore du corps visible reposant sur des représentations (sonores) imaginaires.

8 La mise en scène de la voix d'opéra appelle à la fois un imaginaire vocal culturellement et esthétiquement codé (une intervocalité sémantique ${ }^{15}$ ), tout en réveillant les fantasmes d'une voix mythique (une intervocalité sémiotique). La voix «muette » de Caruso se base ainsi sur le paradoxe esthétique qui allie le sublime éloignement à l'intimité résonnante. 


\section{La construction cinématographique de la diva muette} 16

Ce paradoxe esthétique qui joint le sublime éloignement à la proximité sensible caractérise pour Roland Barthes la construction et la mise en scène du visage de la diva dans le cinéma muet ${ }^{17}$. L'illusion d'une proximité affective et le sentiment d'une distance sublime se rejoignent dans la construction esthétique du phénomène de la diva, dont l'affinité avec le médium cinématographique est à souligner ${ }^{18}$. « Déesse inaccessible $»^{19}$, la diva peut être considérée comme « une figure de la différence entre l'écran et le public, entre la représentation médiale et la personne privée, entre la réalisation technique et les qualités naturelles et individuelles $"{ }^{20}$. La question qui se pose est de savoir dans quelle mesure la construction esthétique de la diva est liée à la mise en scène de la voix dans le médium muet.

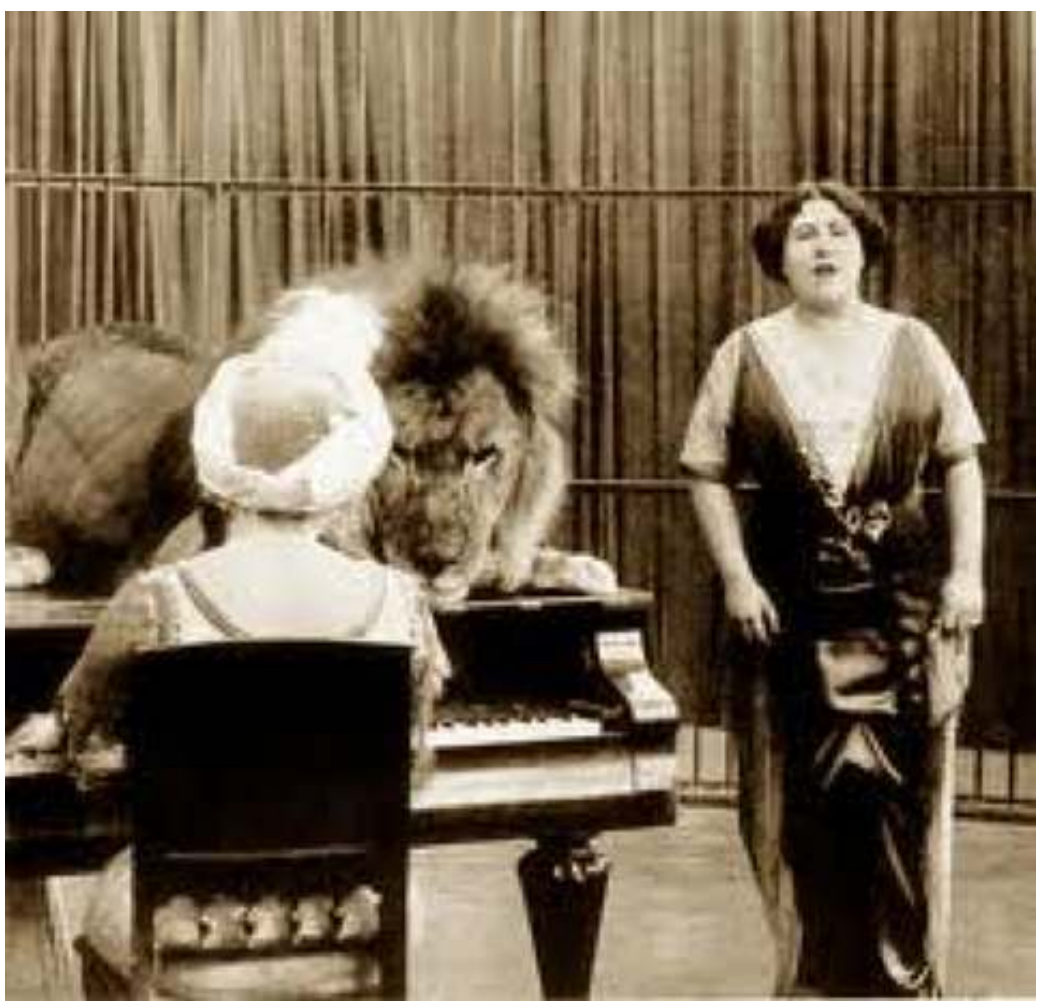

Emmy Destinn dans le film Die Löwenbraut (1913-1914) de Max Obal. Source : isoldes-liebestod.net

En suivant la distinction deleuzienne des deux modalités de l'image-affection, Michael Wedel analyse la mise en scène du visage intensif des films de diva des années 1910 et la force de suggestion esthétique du visage réflexif. Le point de bascule entre le visage intensif et réflexif constitue un moment de suspension du flux narratif. Considéré par Béla Balazs tel un "extrait lyrique de l'ensemble du drame ${ }^{21}$, le visage est au déroulement narratif du film ce que l'air d'opéra est au récitatif, à savoir un moment de réflexivité où la voix se retourne sur elle-même et jouit d'elle-même. Le film Die Löwenbraut (1913-14) de Max Obal, culminant avec la mise en scène de la dimension réflexive de la voix d'Emmy Destinn, présente une scène dans laquelle la diva, enfermée dans une cage à lion, n'a que sa voix pour dompter le fauve. Comme le montre Michael Wedel, il n'est pas étonnant que l'auteur satirique Julius Bauer ait écrit un poème sur la 
voix d'Emmy Destinn depuis la perspective du fauve, car celui-ci reflète la perspective des spectateurs, en tant que corps soumis à la voix, traversés par ces "échos moteurs " ${ }^{22}$ dont parlait Merleau-Ponty pour caractériser la réflexivité vocale. L'imagination vocale engendrée par l'image filmique repose sur le caractère réflexif de la voix qui - et a fortiori dans son exacerbation opératique - noue avec l'expérience esthétique de la voix en tant que phénomène limite entre le propre et l'étranger.

11 En évoquant à dessein publicitaire cette même scène du film, l'affiche publiée dans la revue Motography ${ }^{23}$ souligne l'impassibilité de la diva face aux lions. La puissance de son chant est liée à un dispositif de transcendance et le calme de la chanteuse révèle la composante extatique du chant. Exemplaires en cela sont les grands airs d'opéra où le chanteur se trouve entièrement absorbé par son chant, tandis que le monde qui l'entoure semble disparaitre. Selon Stanley Cavell ${ }^{24}$, la structure "auto-réflexive " ou «narcissique » de la voix s'origine dans la solitude métaphysique de l'homme de sorte que l'opéra prolonge la philosophie et la réflexivité de la voix se comprend comme une « figure de la pensée ». Ce que l'opéra donne à entendre est un cogito in actu que Stanley Cavell nomme le claim, c'est-à-dire la revendication et l'affirmation d'une voix. En supposant que la compréhension phénoménologique et sceptique de la réflexivité de la voix ne s'excluent pas mutuellement, il est possible d'avancer la thèse suivante : en tant que mise en scène de la réflexivité de la voix, la construction esthétique de la diva vise à allier l'expérience esthétique d'un sublime éloignement et d'une proximité sensible avec un moment philosophique (conjugué avec une réflexivité du médium échappant à toute visée narrative), à travers lequel le chant se transforme en cogito revendiquant (claim) son existence - et ce, paradoxalement, malgré son caractère inaudible.

\section{Voix et geste : la transgression du « divisme »}

Puisque l'absence de son ne permet pas aux Divine Diva du cinéma de jouer de leur voix, c'est en traduisant l'expression vocale par des poses, des gestes emphatiques que le " corps excessif» du "divisme ${ }^{25}$ rejoint l'acmé de l'opéra ${ }^{26}$. Soutenant cette thèse, Michèle Lagny identifie les procédés filmiques propres à magnifier la beauté physique, les mimiques expressives et la gestuelle compensatrice de la voix (mise en scène des jeux de regards, gros plans, jeux d'éclairage). La construction cinématographique de la figure de la Diva s'effectue autour du «lieu » principal du corps qu'est la gorge d'où s'origine la voix, articulant le tout du corps avec la valeur symbolique et expressive du visage ${ }^{27}$. Est-ce que le corps de la femme sublime, magnifié dans sa posture et sa gestuelle, mis en valeur par le cadrage et l'éclairage, ne serait, comme l'affirme Michèle Lagny, que " prothèse $»^{28}$ d'une voix, "comme si, dans ses représentations, la femme ne pouvait être à la fois un corps et une voix ${ }^{29}$ ? Sous-entendant ainsi le discours critique des théories féministes du cinéma, la voix restée dans le giron du corps participerait à l'enfermement de la femme dans l'image. Kaja Silverman, dans son analyse de la dimension acoustique du film, définit la position de la voix (féminine, maternelle) du côté de la totalité de l'Imaginaire, emprisonnée dans l'image et opposée/exposée au regard masculin ${ }^{30}$. Ainsi la voix et le corps de la femme sont pris dans la diégèse, enfermés dans l'univers filmique, tandis qu'il revient au sujet masculin de jouir de la distance du regard et d'une extériorité discursive, se positionnant du côté de la maîtrise de la langue, du pouvoir de la vision et de l'écoute. Dans sa critique d'une conception anhistorique et structurelle des relations entre les genres visant la théorie du cinéma d'inspiration psychanalytique, Heide Schlüpmann 
démontre le rôle actif des femmes dans le cinéma des premiers temps, l'(auto-)mise en scène de l'image de la femme (par exemple Asta Nielsen) et affirme le pouvoir du cinéma des premiers temps à s'opposer aux relations de genre organisées autour de la structure patriarcale ${ }^{31}$. À partir d'une analyse du jeu actorial très physique et pleinement engagé des chanteuses Geraldine Farrar et Mary Garden, Mary Simonson envisage leur rôle dans la construction d'une nouvelle image de la femme ${ }^{32}$ : la diva "muette ", plutôt que de remplacer la voix par l'expression corporelle, noue le corps à la voix en transposant le moment de "crise vocale " ${ }^{33}$ (tel que l'entend Wayne Koestenbaum) à l'écran. Pour Koestenbaum, l'attraction de la voix d'opéra ne repose pas uniquement sur les caractéristiques de la voix elle-même mais sur le jeu avec les limites, avec le risque lié à leur franchissement, avec la perte de la voix. C'est ce jeu des limites - celles du chant et de l'expression vocale - que la diva introduit dans le cinéma muet. Elles portent au cinéma la croyance en un art exprimant le pouvoir du silence ${ }^{34}$ comme un moment de "crise vocale », c'est-à-dire un moment de liminarité ${ }^{35}$ qui dérange les oppositions binaires et les structures établies.

\section{Image et voix : le fantôme de l'opéra}

13 L'opéra porte les limites de la voix jusqu'au silence ${ }^{36}$ en interrogeant également les liens entre l'audible et le visible. La relation de la voix au silence touche à la question de l'image comme le rappelle le lien originaire de l'opéra avec le mythe d'Orphée. Grâce au pouvoir de son chant, Orphée est autorisé à passer dans l'autre monde pour ramener Eurydice à la vie. Cependant le chant ne lui permet qu'une victoire momentanée sur la mort. Incapable de soutenir le silence d'Eurydice, son regard annule la puissance du chant. Si la technique du cinéma parlant a cherché à ignorer l'impossibilité énoncée par le mythe en alliant optiquement le son à l'image au moment de la résurrection cinématographique des morts projetés à l'écran, le cinéma muet est traversé par la thématisation de cette impossibilité comme en témoigne la reprise du mythe d'Orphée dans le Fantôme de l'opéra (1925) de Rupert Julian. 

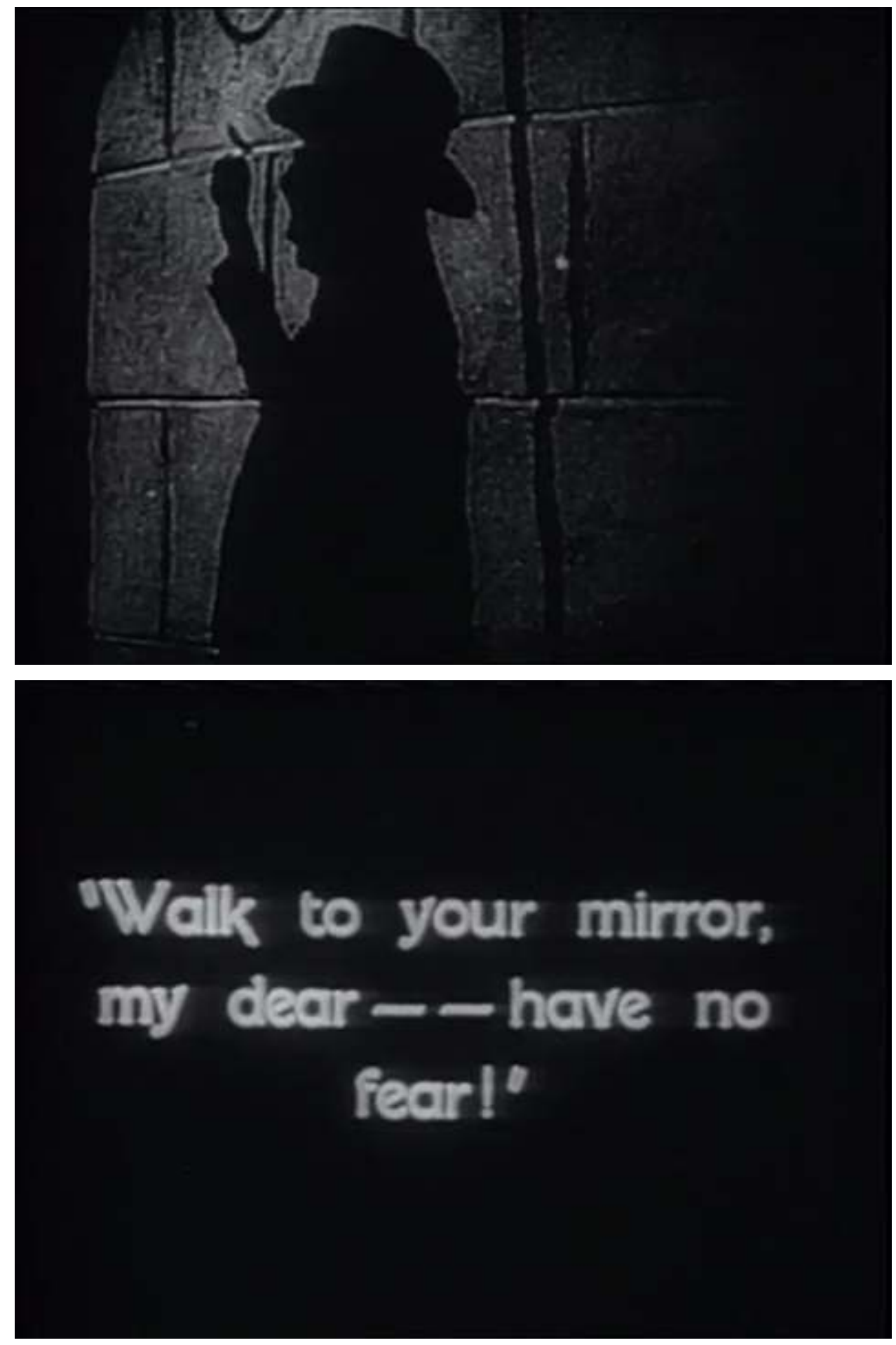


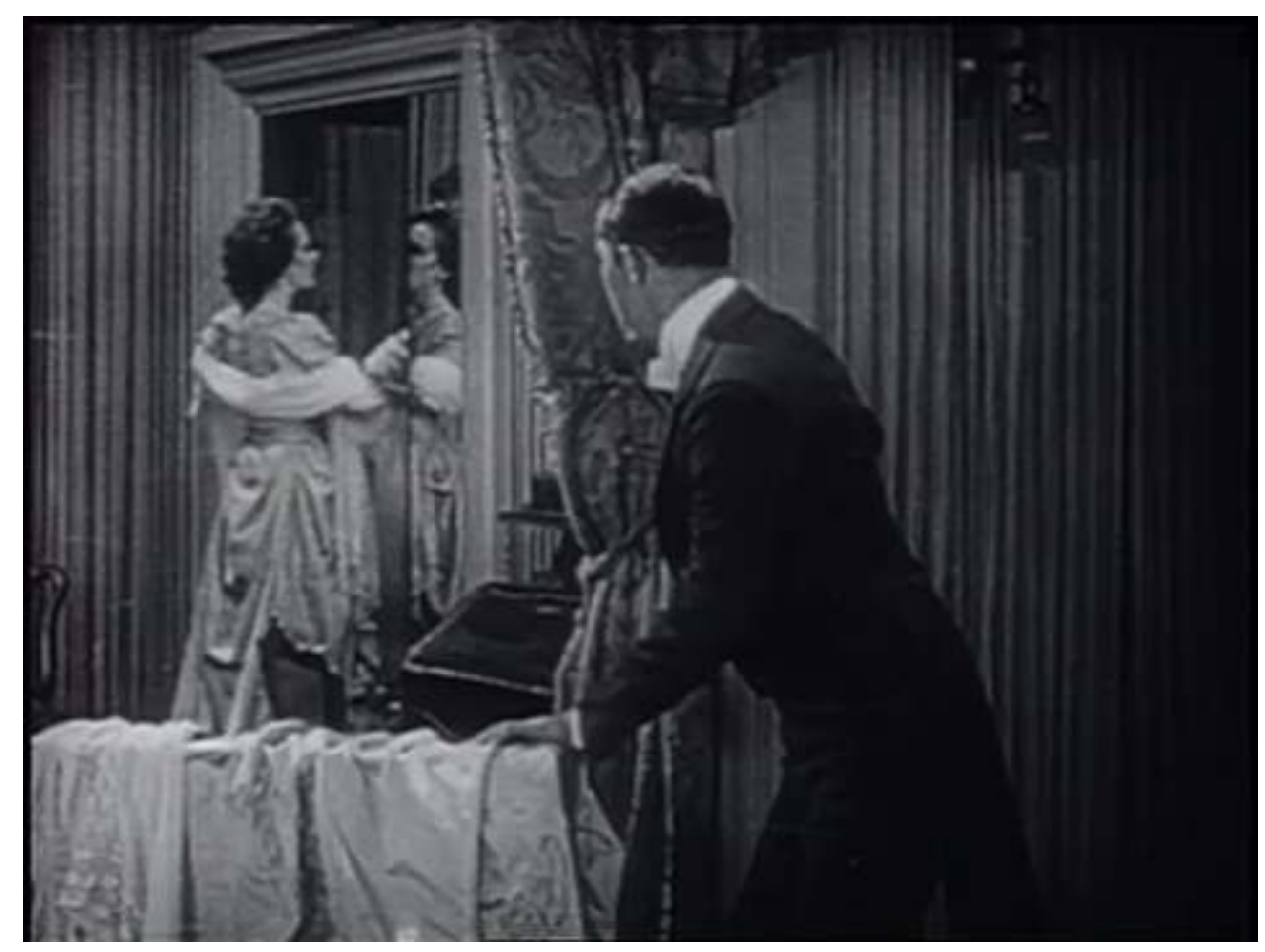

THE PHANTOM OF THE OPERA DE RUPERT JULIAN (1925)

14 Après avoir traversé le miroir pour rejoindre la voix du fantôme, la Prima Donna (Christine) ne peut résister à la tentation de soulever le masque du fantôme (Erik). Comme leur relation ne peut être maintenue à travers la vision, le pouvoir de la voix du fantôme est rompu et Christine perd la sienne puisqu'elle ne chante qu'aussi longtemps que celle d'Erik la hante. Réfléchissant le rapport du chant à l'image, la voix du fantôme de l'opéra apparait comme l'autre face du miroir : Christine, attirée par la voix d'Erik, traverse sa propre image spéculaire. En suivant certaines interprétations et théories psychanalytiques de la voix, l'attirance pour la voix du Fantôme renverrait à l'objet perdu et à l'indivision première qui précède le regard au stade du miroir (Lacan) ${ }^{37}$. L'entrée dans le langage signifie la perte définitive de la voix mythique. Celle-ci peut être approchée tout au plus à travers le silence et le cri - les ressorts centraux de la jouissance pour l'amateur d'opéra selon Michel Poizat ${ }^{38}$. Si un tel «fantôme » habite le film muet, la voix, selon Michal Grover-Friedlander, tend irrémédiablement vers le cri et s'exprime visuellement par des distorsions "voco-visuelles" 39. L'image expressionniste de difformité, d'horreur et les effets de distorsions d'image rendent le fantôme plus profondément opératique que la Prima Donna. Les distorsions « voco-visuelles » seraient analogues aux cris de mort desémentisés ou à la jouissance qui trouent le langage.

15 Selon Slavoj Zizek, « une image peut apparaître comme le tenant-lieu d'un son qui ne résonne pas encore mais reste coincé dans la gorge $»^{40}$. La voix restée en travers de la gorge appartient au domaine du secret, du non-dit, du non-exprimable. L'image tient lieu de cette voix empêchée dont le manque convie à l'imagination. En suivant la conception métapsychologique du fantôme énoncée par Nicolas Abraham ${ }^{41}$, celui-ci ne se limite pas à l'inconnu et à l'indicible mais se constitue comme le produit de la rencontre entre les trouées et les échappées d'un récit, d'une image, d'un film et la faculté d'imagination. Ainsi, la fiction et l'imagination donnent contour à ces trouées. Non seulement Erik est à l'origine de l'opératique d'un point de vue narratif (permettant à Christine d'incarner la 
Prima Donna sur scène en lui soufflant la voix sublime du rôle de Marguerite), mais il est plus essentiellement encore à l'origine de l'opératique lui-même dans le film : en tant que fantôme, il incarne la force de la voix dans le cinéma muet en donnant contour au manque. Mais ce fantôme cinématographique est fragile : pris en chasse, il succombe aux dernières minutes du film laissant apparaitre Christine, heureuse aux côtés de son mari, sa voix sublime de Prima Donna sacrifiée à sa nouvelle vie de femme mariée. Avec la mort d'Erik, c'est tout le fantastique, la magie et les excès de l'opéra qui cèdent leur place au réalisme et à la normalité vocale, pressentant peut-être, deux ans avant la première du film The Jazz Singer la vulnérabilité du fantôme face à l'industrie cinématographique et à la révolution esthétique, technique du parlant.

\section{Le chant entravé de Greta Garbo}

Racontant une histoire d'amour et d'espionnage entre un officier autrichien (Conrad Nagel) et un agent russe (Greta Garbo), le film The Mysterious Lady (1928) développe autour d'un air d'opéra l'essentiel de son axe narratif. Les amants (Karl et Tania) rejouent à plusieurs moments clefs de l'intrigue cet air qui évoque leur première rencontre lors d'une représentation de Tosca, et qui, tel un leitmotiv, accompagne les déboires de leur relation amoureuse. Dans un second temps du film, une rencontre inopinée conduit les anciens amants à rejouer «leur » air d'opéra. Le chant de Tania éveille chez le jeune officier qui l'accompagne au piano des souvenirs tellement douloureux qu'une pulsion d'étranglement le traverse. Se dédoublant tel un spectre, Karl se lève et plaque sa main sur la bouche de Tania. Le procédé filmique de la surimpression permet de présenter Garbo en train de chanter tout en figurant son chant entravé.

Ne pouvant rendre visible la voix qu'en tant qu'inaudible, le cinéma muet figure celle-ci sous forme d'un inaudible médiatisé $e^{42}$ selon une logique proche de la trace. Par-delà la mise en scène de l'écoute insoutenable de Karl et la figuration paradoxale de la puissance de la voix de Tania doublée de son amuïssement, c'est l'image filmique elle-même qui devient opératique en cela qu'elle révèle la puissance de la voix fantomatique du muet qui allie les deux extrêmes : l'image cinématographique transpose visuellement les caractéristiques de la voix d'opéra qui joint l'excès de voix à sa dissolution. 


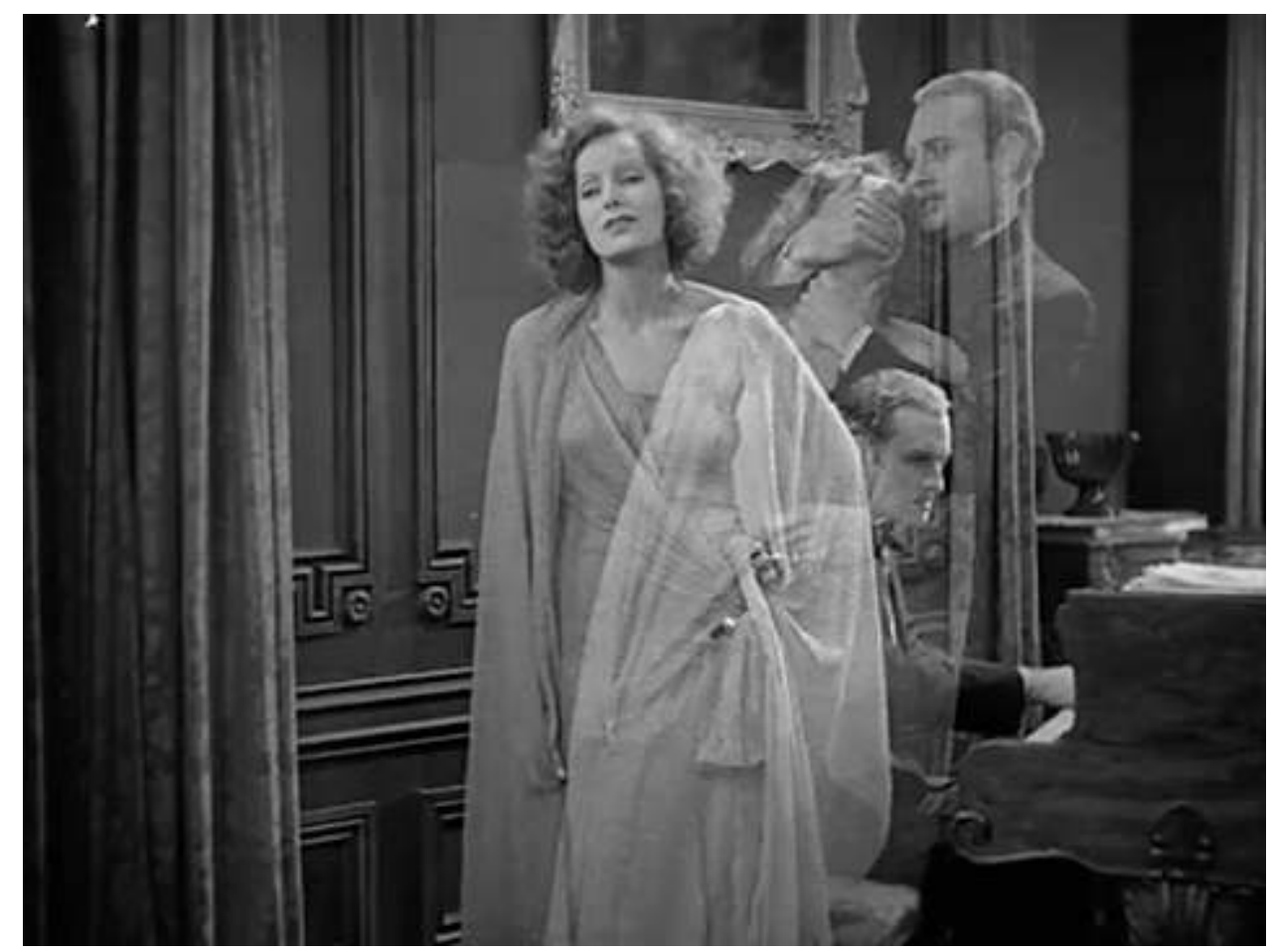

THE MYSTERIOUS LADY DE FRED NIBLO (1928)

\section{Conclusion - Le moment orphique du cinéma muet}

19 L'attirance du cinéma pour l'opéra révèle le moment orphique du muet, comme un moment de transgression du visible et de l'audible, de la vie et de la mort. Le mythe d'Orphée raconte l'impossibilité du chant, essentiellement éphémère, à conserver son acquis : en se retournant sur Eurydice, le regard fige l'instant en une image, et renvoie la bien-aimée au royaume des morts. Cette tension entre la temporalité du chant et de l'image se trouve exacerbée dans le médium cinématographique. Ainsi la dimension orphique du cinéma muet révèle-t-elle la tension entre les images figées et la force du chant qui tente d'infuser la vie dans la mort, entre l'écoute et la vision : image et voix cherchent constamment à se rejoindre sans jamais entièrement s'atteindre.

Comme puissance de transgression entre la vie et la mort, le moment orphique du cinéma muet ouvre à une dimension temporelle porteuse de différance. Alexander Kluge insère dans son film Die Macht der Gefühle (1983) la « citation » filmique d'une adaptation d'Aïda de l'époque du muet, à travers laquelle il pointe le lieu où la mémoire médiatisée, les émotions et les souvenirs individuels se rejoignent. Telle une survivance warburgienne ${ }^{43}$, la visualisation de la voix laisse filtrer les affects passés. À la fois présente et non présente, elle surgit entre singularité et multiplicité pour hanter (au sens derridien) ${ }^{44}$ la scène filmique et réveiller les fantasmes de voix. 


\section{BIBLIOGRAPHIE}

ABRAHAM Nicolas, TÖRÖK Maria, L'écorce et le noyau, Paris, Aubier Flammarion, 1978.

ADORNO Theodor W., Musikalische Schriften I-III, Frankfurt/Main, Suhrkamp Verlag, 2003.

BALAZS Béla, Der sichtbare Mensch oder die Kultur des Films, Frankfurt/Main, Suhrkamp Verlag, 2001

BARTHES Roland, Mythologies, Paris, Seuil, 1957.

CAVELL Stanley, Un ton pour la philosophie, Paris, Bayard, 2003.

COWGILL Rachel, PORISS Hillary (dir.), The Arts of the Prima Donna in the long Nineteenth Century, Oxford Press, 2012.

DERRIDA Jacques, « Fantômes du cinéma », Cahiers du cinéma, avril 2001.

GROVER-FRIEDLANDER Michal, Vocal Apparitions : The Attraction of cinema to opera, Princeton University Press, 2005.

HAMON-SIRÉJOLS Christine, SURGERS Anne (dir.), Theater: sound space, visual space, Presses universitaires de Lyon, 2003.

KERN Doris, NESSEL Sabine (dir.), Unerhörte Erfahrung, Texte zum kino, Festschrift für Heide Schlüpmann zum Geburtstag, Basel/ Frankfurt/Main, Stroemfeld Verlag, 2008.

KOLESCH Doris, KRÄMER Sybille (dir.), Stimme, Annäherung an ein Phänomen, Suhrkamp, Frankfurt/Main, 2006.

MAULE Rosann (dir.), CinéMAS, 16/n.1, Femmes et cinéma muet : nouvelles problématiques, nouvelles méthodologies, colloque de Montréal, Concordia, 2-6 juin 2004.

MERLEAU-PONTY Maurice, Le visible et l'invisible, Paris, Gallimard, 1964.

PAECH Joachim, Der Bewegung einer Linie folgen... Schriften zum Film, Berlin, Vorwerk 8, 2002.

POIZAT Michel, L'opéra ou le cri de l'ange, Essai sur la jouissance de l'amateur d'opéra, Paris, Ed. Metailie, 2001.

SCHLÜPMANN Heide, Unheimlichkeit des Blicks, das Drama des frühen deutschen Kinos, Frankfurt/ Main, 1990.

\section{Filmographie:}

GAD Urban, Die Filmprimadonna, 1913.

JOSÉ Edward, My Cousin, 1918.

JULIAN Rupert, The Phantom of the Opera, 1925.

KLUGE Alexander, Die Macht der Gefühle, 1983.

NIBLO Fred, The Mysterious Lady, 1928.

OBAL Max, Die Löwenbraut, 1913-14. 


\section{NOTES}

1. «GERALDINE FARRAR - sa voix chante encore dans le cœur des Russes, des Teutons, des Francs, des Anglais - aux oreilles emplies de sons plus rudes - joue à présent dans un opéra muet des studios Lasky à Hollywood en Californie. La Farrar sans voix! La Mona Lisa sans son sourire, un stradivarius sans ses cordes, des enfants d'un institut de sourds-muets se produisant au théâtre »./ «GERALDINE FARRAR- her voice is still singing in the hearts of Russ, Teuton, Frank and Englishman, though ruder sounds fill their ears- is now doing silent opera at the Lasky studios in Hollywood, California. Farrar voiceless! The Mona Lisa without her smile ; a Stradivarius without its strings ; children of a deaf and dumb institute at play » dans Photoplay Magazine, août 1915, p. 42. Cité par Mary Simonson, "Screening the Diva », dans Rachel Cowgill, Hillary Poriss (dir.), The Arts of the Prima Donna in the long Nineteenth Century, Oxford Press, 2012, p. 83.

2. Stanley Cavell, Un ton pour la philosophie, Paris Bayard, 2003, p. 195.

3. Issu du romantisme, le concept d' « œuvre d'art totale » est développé par Richard Wagner au milieu du XIXe siècle (L'Éuvre d'art du futur, 1849 et Opéra et drame, 1851). Prenant pour modèle idéal le théâtre antique, l'œuvre d'art totale vise la réconciliation de la danse, de la musique et de la poésie, des «trois sœurs jumelles» qui se complètent mutuellement dans le drame musical. L'unité des arts est supposée refléter l'unité de la vie.

Ricciotto Canudo voit dans le " septième art » la promesse de réalisation d'une « totale synthèse " à laquelle ont aspirés tous les autres arts.

4. Pour une filmographie détaillée voir Wlaschin, Ken, Encyclopedia of Opera on Screen, New Haven, Conn.,Yale University Press, 2004.

5. Dans le contexte germanophone, ce genre est discuté sous des noms aussi divers qu'Opernfilme, Filmoper, Kinooper, Lichtspieloper. Dans le domaine anglophone, il est question de soundless opera. En France, le terme de symphonie visuelle se rapporte plus globalement à l'analogie musicale très discutée dans le cinéma français des années 20. Oliver Huck, Das musikalische Drama im "Stummfilm ". Oper, Tonbild und Musik im Film d'art, Hildesheim, Olms, 2012. E. H. Bierstadt, « Opera in Moving Pictures », Opera Magazine, Octobre 1915, p.30.

" Musicalisme », Dictionnaire du cinéma français des années 20, François Albera et Jean A. Gili (dir.), 1895. Mille huit cent quatre-vingt-quinze [En ligne], 33 | 2001: http://1895.revues.org/97.

6. Cette généralisation mériterait un développement plus détaillé et différencié de l'histoire du cinéma et de l'opéra, dont il ne peut être question ici.

7. L'opéra en France ne jouirait pas du même statut au sein du genre dramatique que l'opéra en Allemagne. Selon Olivier Huck notamment (op.cit., p.1), c'est avant tout l'emprunt de l'argument dramatique qui justifie, dans la tradition française, l'analogie entre cinéma et opéra. Les films accompagnés d'une musique originale ne sont pas tant comparés à l'opéra qu'au poème symphonique, au ballet, ou à la pantomime.

8. Thomas Elsaesser, Filmgeschichte und frühes Kino: Archäologie eines Medienwandels, edition text +kritik, Munich, 2002, p. 178-179.

9. Il s'agit des procédés de synchronisation du cinématographe et du phonographe comme le chronophone Gaumont employé pour les phonoscènes d'Alice Guy, le chronomégaphone ou encore le« Biophon » mis au point par Oskar Messter en Allemagne.

10. A la suite d'Immacolata Amodeo, je comprends l'opératique (das Opernhafte) comme une qualité transmédiale. Immacolata Amodeo, Das Opernhafte, Eine Studie zum "gusto melodrammatico" in Italien und Europa, Bielefeld, Transcript Verlag, 2007. 
11. Un plan (en général un gros-plan) qui montre la réaction d'un ou plusieurs personnages par rapport au plan précédent. Dans My Cousin, un chien hurle, des voisins se bouchent les oreilles en réaction au chant de Tommasso.

12. «Vesti la giubba e la faccia infarina...» / " Revêts la tunique, enfarine-toi le visage...»

13. «Si les gestes eux-mêmes, que les chanteurs puisent en quelque sorte dans un fonds, représentent déjà une part du costume, la voix, elle, est entièrement costume, que l'homme naturel revêt pour ainsi dire sitôt qu'il monte sur la scène de l'opéra » / " Sind schon die Gesten der Sänger selbst, die sie oft gleichwie aus dem Fundus mitbringen, ein Stück Kostüm, so ist es vollends ihre Stimme, die der natürliche Mensch, sobald er die Opernbühne betritt, gewissermassen anlegt ", Theodor W. Adorno, Musikalische Schriften I-III, Frankfurt/Main, Suhrkamp Verlag, 2003, p.25.

14. J'entends à ce propos la représentation filmique du personnage conjuguée à sa représentation plastique. Ainsi se retrouve au centre de l'intrigue le buste de Caroli réalisé par Tommasso.

15. Je renvoie à la distinction d'Helga Finter entre une première intervocalité sémiotique (selon le terme de Kristeva) relevant de la psychogénèse de la voix et une intervocalité sémantique liée aux codifications sociales, culturelles et psychologiques. Helga Finter, "Mime de Voix, mime de corps : l'intervocalité sur scène» dans International Federation for Theatre Research, Christine Hamon-Siréjols, Anne Surgers (dir.), Theater: sound space, visual space, Presses universitaires de Lyon, 2003, pp. 80-81.

16. Je reprends ici pour une majeure partie l'argumentation de Michael Wedel, «Wenn eine Dame .... Löwen im Käfig hält », dans Doris Kern, Sabine Nessel (dir.), Unerhörte Erfahrung, Texte zum kino, Festschrift für Heide Schlüpmann zum Geburtstag, Basel/ Frankfurt/Main, Stroemfeld Verlag, 2008, pp.158-179.

17. Roland Barthes, Mythologies, Paris, Seuil, 1957, p. 77.

18. Heide Schlüpmann, Der Auftritt der Diva, http://www.kinothek-asta-nielsen.de/pdf/diva_ schluepmann.pdf, p.4, consulté le 16 avril 2014.

19. Claudia Balk, Theatergöttinen. Inszenierte Weiblichkeit. Clara Ziegler, Sarah Bernhardt, Eleonore Duse , Frankfurt/Main 1994, p. 75.

20. Michael Wedel, op.cit., p.162.

21. «Ein lyrisches Extrakt des ganzen Dramas »,Béla Balazs, Der sichtbare Mensch oder die Kultur des Films, Frankfurt/Main, Suhrkamp Verlag, 2001, p.48.

22. Maurice Merleau-Ponty, Le visible et l'invisible, Paris, Gallimard, 1964, p. 190.

23. «Featuring Mme Emmy Destinn, the great Prima Donna, singing a part of Mignon in a cage containing fourteen lions, one of which was within two or three feet of the singer all the time. The nerve she shows is almost hair raising. » dans Motography (janvier-juin 1914), Vol.IX, Nr. 13, p. 4.

24. Stanley Cavell, Un ton pour la philosophie, Paris, Bayard, 2003, pp. 185-236.

25. Francesco Pitassio, Ombre silenziose, Pasian di Prato, Campanotto Editore, 2002, p. 196-197. Cité par Michèle Lagny, "Avec ou sans voix, la femme défaite », CinéMAS, 16/n.1, Femmes et cinéma muet : nouvelles problématiques, nouvelles méthodologies, Rosann Maule (dir.), colloque de Montréal, Concordia, 2-6 juin 2004, p. 127.

26. Analyse partagée par nombre d'historiens et théoriciens du cinéma tels que Michele Canosa, Francesco Pitasso, Michèle Lagny.

27. Michèle Lagny, op.cit., p.129-132.

28. Michèle Lagny, op.cit., p.134.

29. Michèle Lagny, op.cit., p. 134.

30. Kaja Silverman: The Acoustic Mirror. The Female Voice in Psychoanalysis and Cinema, Indianapolis/Bloomington, 1988.

31. Heide Schlüpmann, Unheimlichkeit des Blicks, das Drama des frühen deutschen Kinos, Frankfurt/ Main, 1990, p. 308. 
32. Mary Simonson, "Screening the Diva ", dans Rachel Cowgill, Hillary Poriss (dir.), The Arts of the Prima Donna in the long Nineteenth Century, Oxford Press, 2012, p. 95.

33. « Vocal crisis ». Wayne Koestenbaum désigne par ce terme : « une forme de communication. Cela nous dit que l'opéra est un art de l'interruption, de la rupture et du danger physique. Dans la crise vocale, le chanteur paie le prix de sa sublimité » / «a form of communication. It tells us that opera is an art of interruption, rupture, and bodily danger. The singer in vocal crisis has been punished for sublimity ». Cité par Mary Simonson, ibid., p. 96.

34. Michal Grover-Friedlander, Vocal Apparitions : The Attraction of cinema to opera, Princeton University Press, 2005, p. 21.

35. Développé par l'ethnologue Arnold Van Gennep, le concept de liminarité désigne une phase des rites de passage pendant laquelle l'individu se situe entre son ancien statut et le nouveau.

36. «Il ne nous semble pas absurde en effet d'envisager l'histoire de l'opéra comme un long cheminement qui partirait de la parole, chantée au plus près ou de la prosodie de la langue, et qui aboutirait, via le chant de plus en plus détaché de la parole et de plus en plus tendu vers l'aigu au cri pur. Ce cri, non musicalisé, c'est-à-dire non inscrit sur la portée, non inséré de ce fait dans le discours musical mais explicitement voulu par le compositeur comme pur effet vocal, ce cri, présentifierait, dans le rapport qu'il entretient avec le silence [...] ce point limite de la quête de l'objet-voix où le frisson du plaisir bascule dans le frisson d'horreur [...] », Michel Poizat, L'opéra ou le cri de l'ange, Essai sur la jouissance de l'amateur d'opéra, Paris, Ed. Metailie, 2001, p.65.

37. Jacques Lacan, «Le Stade du miroir comme formateur de la fonction du Je : telle qu'elle nous est révélée dans l'expérience psychanalytique », Revue française de psychanalyse, octobre 1949, p. 449-455.

38. Michel Poizat, op. cit, pp. 15-63.

39. Michal Grover-Friedlander, op.cit., p.156.

40. "An image can emerge as the placeholder for a sound that doesn't yet resonate but remains stuck in the throat ", Slavoj Zizek, «I Hear You with My Eyes ", dans Renata Salecl, Slavoj Zizek (dir.), Gaze and Voice as Love Objects, Duke University Press, 1996, p. 93.

41. Nicolas Abraham, Maria Török, L'écorce et le noyau, Paris, Aubier Flammarion, 1978, p. 427.

42. Je remplace ici l'expression d'« invisible médiatisé » ( "mediatisierte Unsichtbarkeit») par celle d'« inaudible médiatisé », Joachim Paech, Der Bewegung einer Linie folgen... Schriften zum Film, Berlin, Vorwerk 8, 2002, p. 118.

43. Développée par l'historien de l'art Aby Warburg, la notion de « survivance » ( Nachleben ») désigne la capacité de revenance de l'image, de survivance des formes et de la transmission d'un pathos à travers le temps. Voir à ce propos Sigrid Weigel, «Pathosformel und die Oper : Die Bedeutung des Musiktheaters für Aby Warburgs Konzept der Pathosformel », KulturPoetik, Bd.6, H. 2, 2006, pp. 250-253.

44. "L'expérience cinématographique appartient, de part en part, à la spectralité, que je relie à tout ce qu'on a pu dire du spectre en psychanalyse - ou à la nature même de la trace ", Jacques Derrida, « Fantômes du cinéma », Cahiers du cinéma, avril 2001, p. 77.

\section{RÉSUMÉS}

Cet article se propose d'étudier la mise en scène de la voix lyrique dans le cinéma muet. Il s'agit d'interroger la représentation visuelle du pouvoir de la voix, le paradoxe esthétique que 
constitue la figure de la diva au cinéma muet, le rôle de l'évocation de la voix lyrique au niveau de la réception filmique ainsi que sa dimension réflexive dans le médium muet. Se cristallisant de manière paradigmatique autour de la construction esthétique de la figure de la diva "muette ", c'est autour de l'élément vocal que se nouent les points de rencontre entre cinéma muet et opéra. La thèse soutenue dans cet article énonce que c'est précisément la "mutité » de la voix qui confère son caractère "opératique " au cinéma. Le moment "orphique » du cinéma muet se révèle alors comme un moment de transgression du visible et de l'audible, de la vie et de la mort, transcendant les époques dans un mouvement de survivance où la mémoire médiatisée, les émotions et les souvenirs individuels se rejoignent.

This paper studies the strategies of staging lyrical voice in silent movie. It scrutinizes the visual representation of the voice's particular force, the esthetic paradox manifesting itself through the figure of the diva in silent film, the lyrical voice's evocative power during the reception process and its reflexive dimension within the silent medium. Crystallizing paradigmatically around the esthetic construct of the 'silent' diva it is the soundless voice in which silent movie and opera meet. Thus the argument put forward in this paper is that it is precisely the 'silentness' of the voice that confers a specific operatic character to the movies. The 'orphic' moment of silent film turns out to be a moment of transgression from the visible to the audible, from life to death, transcending historical linearity toward an instant of 'survivance' in which mediated memory, emotions and individual souvenirs converge.

\section{INDEX}

Palabras claves : Opéra, Cinéma muet, Voix, Intervocalité, Intermédialité, Fantôme, Orphée Schlüsselwörter : Opéra, Cinéma muet, Voix, Intervocalité, Intermédialité, Fantôme, Orphée Keywords : Opéra, Cinéma muet, Voix, Intervocalité, Intermédialité, Fantôme, Orphée Mots-clés : Opéra, Cinéma muet, Voix, Intervocalité, Intermédialité, Fantôme, Orphée

\section{AUTEUR}

\section{SOPHIE HERR}

Doctorante à l'Université Libre de Berlin, Sophie Herr prépare une thèse sur la voix dans le cinéma muet sous la direction de Doris Kolesch et Gertrud Koch. Elle est actuellement membreboursière de l'International Research Training Group InterArt de l'Université Libre de Berlin. 